\title{
The impact of magnetic resonance imaging in the assessment of iron overload in heart and liver in transfusion-dependent thalassemic children: Minia experience
}

\author{
Ashraf M. El Sherif ${ }^{*}$, Ahmed S. Ibrahim², Mohamed A. Elsayed ${ }^{1}$, Ahmed S. Abdelhakim and Ahlam M. Ismail ${ }^{3}$
}

\begin{abstract}
Background: Thalassemia is the most prevalent single-gene disorder. Myocardial and hepatic iron depositions lead to complications and eventually death. We aimed to assess the diagnostic efficacy of magnetic resonance imaging T2* (MRI T2*) in quantifying iron overload in liver and heart in transfusion-dependent B-thalassemia major (TDT) children.

Methods: Prospective clinical study was carried on sixty children diagnosed with TDT. All of them underwent laboratory investigations, including CBC, serum iron, and ferritin levels. MRI T2* of the heart and liver was carried out to measure the iron overload and estimate the left ventricular ejection fraction (LVEF).

Results: Thirty-eight males and 22 females with TDT with a mean age of 13.23 years were included. Twenty cases (33.3\%) had severe liver iron overload, while 36 (60\%) had normal cardiac iron. There was a moderate significant negative association between hepatic and cardiac iron deposition $(P=0.03)$. All cases with severe cardiac iron overload had impaired LVEF below 56\%. A non-significant positive association was noticed between cardiac iron deposition and LVEF in T2* $(P=0.08)$. A moderate negative significant association was detected between hepatic iron deposition and serum ferritin, while a fair negative significant association was found between serum ferritin and cardiac iron deposition with $P$ values of 0.04 and 0.02 , respectively.
\end{abstract}

Conclusion: MRIT2* is the gold standard for monitoring and follow-up of iron overload in the heart and liver. It should be routinely performed in all TDT children as liver iron, and serum ferritin do not reflect cardiac iron overload.

Keywords: TDT, MRI T2*, Iron overload, Liver, Heart, Children

\section{Background}

Thalassemia is the most prevalent single-gene disorder globally, with around 94 million heterozygous for beta-thalassemia and 60,000 homozygotes born every year. It is a hereditary hemolytic disease resulting in abnormal hemoglobin synthesis and is common among

\footnotetext{
*Correspondence: ashraf5566@hotmail.com

${ }^{1}$ Department of Radiology, Faculty of Medicine, Minia Maternity and Children University Hospital, Minia University, Minia, Egypt Full list of author information is available at the end of the article
}

Mediterranean populations [1]. Three types of thalassemia are classified according to their clinical severity: major, intermediate, and minor. The most serious type is thalassemia major (TM). Individuals with TM have chronic hemolytic anemia, which often needs lifelong transfusion therapy and can result in tissue iron overload. Cardiovascular complications resulting from myocardial iron overload continue to be a major reason for morbidity and death in those cases [2]. Even though survivability is increasing in cohorts of cases treated with chelation therapy at an early age [3], myocardial siderosis 
with subsequent heart failure continues to be major reason for mortality $(50-70 \%)$ in thalassemia major cases. This disease manifests itself at an alarmingly early age with about $15-50 \%$ of cases dying before the age of 35 [3, 4]. Once clinically evident heart failure is apparent and catastrophic decline in heart function, leading to death may occur fast. Prediction of heart failure depends on actual iron loading measurements; time-averaged serum ferritin $>2500 \mu \mathrm{g} / \mathrm{L}[5,6]$ and hepatic iron concentration $>15 \mathrm{mg} / \mathrm{kg}$ [7]. Nevertheless, the persistently high death rate from heart failure suggests that high-risk cases are not recognized in time for intervention strategy. Ventricular function assessment, like changes in ejection fraction over time, has also been suggested in thalassemia. However, this method detects cases relatively late [8], and the impairment may be concealed due to supranormal left ventricular performance in thalassemia cases without myocardial iron overload [9]. Of these methods, the measurement of Magnetic resonance imaging T2* (MRI T2*) has become the most often utilized in the heart due to its ease of integration with heart gating, its speed and robustness, and its sensitivity to iron deposit [10]. Cardiovascular T2* imaging is transferable, with excellent inter-scanner accuracy [11], and exhibits a good association with the invasive method of biopsy. Direct calibration of the heart and liver $\mathrm{T} 2 \%$ values to myocardial and hepatic iron concentrations has been recorded in animals [12] and humans [13].

\section{Objectives}

We targeted to assess cardiac and liver iron overload in transfusion-dependent B thalassemia major (TDT) children using MRI T2*.

\section{Methods}

This prospective clinical study was done in the Radiology department at Minia Maternity and Children University Hospital in Egypt. Sixty children diagnosed with transfusion-dependent beta-thalassemia major (TDT) depending on hematological and clinical evaluation and aged between 5 and 15 years were included in the study. Recruited children were on regular blood transfusion each 2-4 weeks to keep the hemoglobin ( $\mathrm{Hb} \%)$ between 9-11 g/dl. Permission was obtained from the local ethics committee at the Faculty of Medicine, Minia University, and informed consent was collected from the guardians or parents of the children. We excluded thalassemic children younger than five years old, cases with insufficient transfusion to cause hepatic or cardiac siderosis, and cases with a condition incompatible with MRI.

All enrolled children underwent a detailed history taking, including demographic data and clinical characteristics, including age, sex, residence, and duration of the disease. Details of chelation therapy were obtained, including orally administered chelating agents as deferasirox (DFX) and deferiprone (DFP), injectable forms as subcutaneous (SC) or intravenous (IV) desferrioxamine (DFO), compliance to treatment, and regularity of their use. The duration, dose, and type of treatment, whether monotherapy or in combination, were also confirmed. The past history of splenectomy was recorded if it was done. All children were diagnosed and treated according to The Thalassemia Clinical Research Network (TCRN) guidelines [14].

Clinical examination and laboratory investigations were performed, involving kidney and liver function tests, complete blood count, ferritin levels, and serum iron. MRI of the liver and the heart was carried out in all cases.

\section{MRI methodology and image acquisition}

MRI study was done on a 1.5-Tesla MR system (Ingenia; Philips Medical Systems, the Netherlands). ECG and respiratory gating were used with dedicated phased array Torso coil using single breath-hold multi-echo gradient echo sequence as follows: 1 . To begin, localizer images in three orthogonal dimensions were obtained (axial, sagittal, and coronal) SSFP (B-FFE). They are: 1. Fast single-shot scans (fast SSh). 2. Left anterior oblique (LAO) vertical long-axis view. 3. Four-chamber view (P4CH). 4. Multiecho turbo-field echo (mTFE)/fast field echo (FFE) cardiac black blood (BB) short-axis view. 5. Multiecho turbo-field echo (mTFE) Cardiac white blood (WB) short axis. 6. Multiecho turbo/fast field echo (mTFE/m-FFE) Liver FIG (Axial) Imaging field of view. 7. In short-axis view, functional cine images utilizing an ECG-gated segmented $\mathrm{k}$-space breath-hold balanced turbo-field echo (b-TFE) sequence.

Postprocessing was done using region of interest-based measurement to calculate the $\mathrm{T} 2 *$ value of the heart, liver iron concentration (LIC) in $\mathrm{mg} / \mathrm{g}$ dry weight, and left ventricular ejection fraction (LVEF).

\section{Cardiac T2* and LVEF measurements}

Utilizing a single breath-hold ECG-gated multi-echo dark blood method, we recorded a single short-axis midventricular slice (slice thickness: $10 \mathrm{~mm}$, TR: $710 \mathrm{~ms}$, FOV: $400 \times 300 \mathrm{~mm}$, flip angle: $20^{\circ}$, bandwidth: $810 \mathrm{~Hz} / \mathrm{Px}$, matrix: $256 \times 96 \mathrm{~mm}$ ). This $\mathrm{T} 2 *$ series produced a series of eight images with TEs ranging from 1.5 to $17.3 \mathrm{~ms}$ and a gap of $2.3 \mathrm{~ms}$. Next, on each image, a region of interest (ROI) in the ventricular septum was chosen to determine the signal strength.

The end-diastolic volumes (EDV) and end-systolic volumes (ESV) of the left ventricle were determined. By applying standard software analysis techniques, 
endocardial borders were manually outlined at end-systole (ES) and end-diastole (ED). Volumes were calculated using the summation of the disks approach ("Simpson's rule"), which multiplied the sum of all slices by each slice thickness. Following that, the ejection fraction was determined utilizing ESV and EDV. The ejection fraction was determined utilizing a standard CMR sequence, and an EF of less than $56 \%$ was regarded to be suggestive of cardiac dysfunction.

\section{Liver T2* measurements}

The (ROI) was drawn in homogenous liver tissue from an axial mid hepatic slice of the right lobe avoiding bile ducts and blood vessels. The ROI was produced throughout all images, and signal intensity was measured using truncation and offset models (where a constant offset is applied to account for long $\mathrm{T} 2 *$ and noise components). $\mathrm{R} 2 *$ values $(=1000 / \mathrm{T} 2 *)$ were turned into LIC $(\mathrm{mg} / \mathrm{gdw})$ utilizing Garbowski's equation [15]: $\mathrm{LIC}=0.03 \times \mathrm{R} 2$ $* 0.7$

According to calculated $\mathrm{T} 2 \%$, cardiac iron load in cases was classified as acceptable $\left(\mathrm{T} 2^{*}>20 \mathrm{~ms}\right)$, mild ( $2^{*} * 15-20 \mathrm{~ms}$ ), moderate (T2* 10-14 ms), and severe $(\mathrm{T} 2 *<10 \mathrm{~ms})$ myocardial affection [16, 17]. Liver iron concentration (LIC) was classified as acceptable ( $3.5 \mathrm{mg} / \mathrm{g})$ mild $(3.5-7 \mathrm{mg} / \mathrm{g})$, moderate $(7-12 \mathrm{mg} / \mathrm{g})$ and severe (>12 mg/g) [18] (Fig. 1).

\section{Statistical analysis}

The data were analyzed utilizing the SPSS 20.0 statistical package. The Kolmogorov-Smirnov or Shapiro-Wilk tests were utilized to identify the data normality. For quantitative parametric measures, data were recorded as mean \pm standard deviation (SD), minimum and maximum of range, or median and range for quantitative nonparametric measures. For parametric data, the student $t$ test was employed; the Mann-Whitney test was used for nonparametric data. Analysis of variance (ANOVA) was employed for comparison between parametric data. To compare categorical variables, the Chi-square test or fisher's exact were utilized. Pearson's association analysis was used to determine the associations between the parameters. Association coefficients of $r=0-0.24$ were regarded weak, $r=0.25-0.49$ were considered fair, $r=0.5-0.74$ were considered moderate, and $r=0.75-1$ were considered strong. The $P$ value of 0.05 or less was significant.

\section{Results}

Our work was done on 60 children with TDT on longterm blood transfusion. Their ages were between 5 and 15 years, with a mean age of $13.23 \pm 3.01$ years. Thirtyeight cases $(63.3 \%)$ were males, and 22 were females. Table 1 presents the laboratory and clinical data of the cases. Regarding the hepatic iron overload severity according to $\mathrm{T}^{*}$ in this study, the majority $(76.6 \%)$

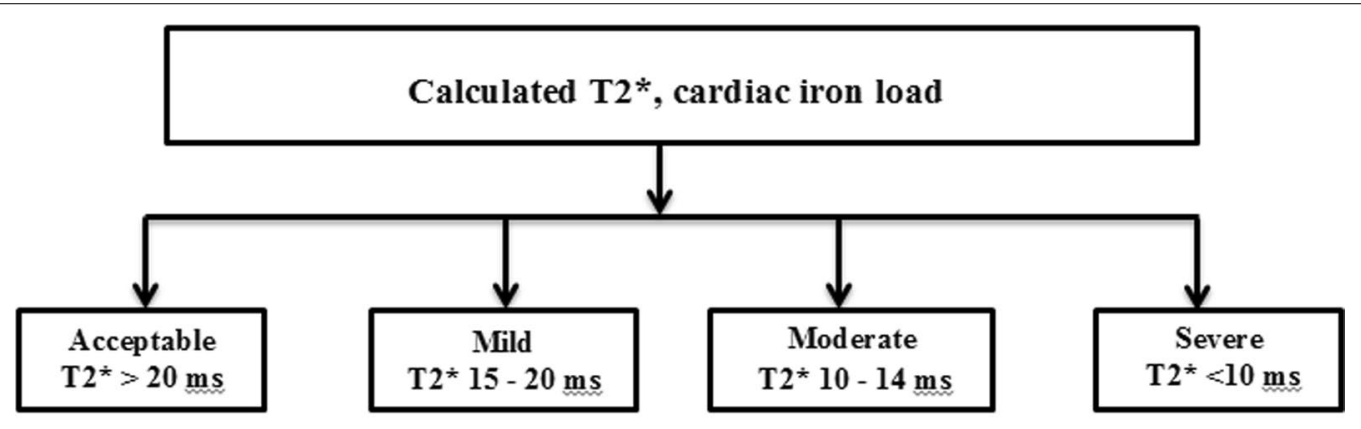

\section{Liver iron concentration (LIC)}

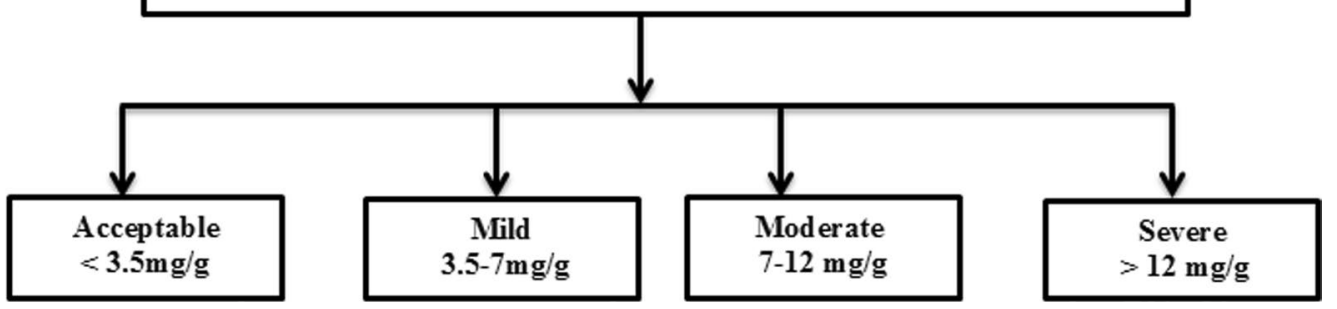

Fig. 1 Calculated T2*, cardiac iron load and liver iron concentration (LIC) in transfusion-dependent beta-thalassemia major children 
Table 1 Clinical characteristics and laboratory data of 60 children with transfusion-dependent beta-thalassemia major (TDT)

\begin{tabular}{ll}
\hline Characteristics of the studied children & Total number $=\mathbf{6 0}$ \\
\hline Age (years) & $5-15(13.23 \pm 3.01)$ \\
Sex & $38(63.3 \%) / 22(36.7 \%)$ \\
Males/females & $18-48(29.73 \pm 4.76)$ \\
Wt. (kg) & $110-160(126.60 \pm 12.4)$ \\
Height (cm) & $4-15(5.88 \pm 3.42)$ \\
Duration of the disease (years) & \\
Iron chelating agents & $32(53.3 \%) / 28(46.7 \%)$ \\
Regular/irregular & \\
Type of chelation therapy & $10(16.6 \%)$ \\
$\quad$ Deferoxamine monotherapy (SC, IV) & $25(41.7 \%)$ \\
Deferasirox monotherapy (Oral) & $6(10 \%)$ \\
Deferiprone monotherapy (Oral) & $19(31.7 \%)$ \\
Deferoxamine (SC,IV) and deferasirox & \\
(Oral) & \\
Splenectomy & $34 / 60(56.6 \%) / 26 / 60(43.4 \%)$ \\
Done/not done & $7-10.5(9.01 \pm 1.2)$ \\
Hb level (gm/dl) & $650-1200(803.68 \pm 261.36)$ \\
Serum iron (mcg/dl) & $1900-6000(2639.47 \pm 835.06)$ \\
Serum ferritin (ng/ ml) &
\end{tabular}

SC subcutaneous, IV intravenous

" Data presented as a range (mean \pm SD)

of the cases had a noticeable liver iron overload (LIC 4-13.4 mg/g). Twenty cases (33.3\%) had severe liver iron overload, 14 cases $(23.3 \%)$ had a moderate overload, and $12(20 \%)$ had a mild overload. In contrast, the majority of study cases, $36(60 \%)$, had normal cardiac iron stores, while $10(16.7 \%), 8(13.3 \%)$, and 6 (10\%) had mild, moderate, and severe cardiac iron deposition, respectively (Table 2). A significant statistical difference was observed between hepatic and cardiac iron deposition, where the frequency of iron deposition was greater in the liver than in the heart, with a $P$ value of 0.02 (Table 2). There was a moderate significant negative association between

Table 2 Iron overload severity in liver and heart according to T2* in children with transfusion-dependent beta-thalassemia major (TDT)

\begin{tabular}{llll}
\hline $\begin{array}{l}\text { Degree of iron } \\
\text { overload }\end{array}$ & $\begin{array}{l}\text { Heart } \\
\text { Cases no (\%)" }\end{array}$ & $\begin{array}{l}\text { Liver } \\
\text { Cases no (\%) }\end{array}$ & P value \\
\hline Normal & $36(60 \%)$ & $14(23.3 \%)$ & $0.02^{*}$ \\
Mild & $10(16.7 \%)$ & $12(20 \%)$ & \\
Moderate & $8(13.3 \%)$ & $14(23.3 \%)$ & \\
Severe & $6(10 \%)$ & $20(33.3 \%)$ & \\
\hline
\end{tabular}

"Number (No) and Percentage (\%) *P value was regarded as statistically significant if $<0.05$ cardiac and hepatic iron deposition $(P=0.03)$. In this study, all cases with severe cardiac iron overload (10\%) had impaired left ventricular ejection fraction less than $56 \%$. A non-significant positive association was detected between ejection fraction (EF) and cardiac iron deposition in $\mathrm{T}_{2} *(P=0.08)$. A moderate negative significant association was observed between hepatic iron deposition and serum ferritin, while there was a fair negative significant association between serum ferritin and cardiac iron deposition with $P$ values of 0.04 and 0.02 , respectively (Table 3) (Figs. 2, 3).

\section{Discussion}

Blood transfusion in cases with $\beta$-thalassemia major results in the deposition of iron in the reticulo-endothelial system (RES) of the heart, liver, and many other organs [19]. Therefore, monitoring cardiac iron overload is very important. Because serum ferritin is an acute-phase protein, its concentrations might be impacted by inflammation, vitamin $C$ levels, infection, chelation therapy, and liver injury [20]. Tissue biopsy, the only method allowing direct and reliable measurements of iron deposition, cannot be routinely used as it is invasive and impacted by the unequal distribution of iron deposition, which may skew results [19]. Magnetic resonance has acquired an important role in monitoring cases with hemosiderosis; this allows evaluating iron quantity and distribution in the various organs in a noninvasive way [21]. The magnetic resonance imaging of the $\mathrm{T} 2 *$ approach can help diagnose myocardial iron overload [22]. Iron deposition produces inhomogeneous magnetic fields and reduces the relaxation variables $\mathrm{T} 1, \mathrm{~T} 2$, and $\mathrm{T} 2 \%$. A cardiac T2*value of $<20 \mathrm{~ms}$ indicates iron overload [23]. Our work was done on 60 cases with thalassemia major that were dependent on long-term blood transfusion. We measured both hepatic and cardiac iron overload in the same session and categorized cases into normal, mild, moderate, and severe iron overload according to their signal intensity and the T2* values using multiple TEs. In our work, we have presented that $40 \%$ of the cases had an abnormal overload of iron in the heart $(\mathrm{T} 2 *<20 \mathrm{~ms})$.

Table 3 Association between certain variables and hepatic iron deposition in 60 children with transfusion-dependent betathalassemia major (TDT)

\begin{tabular}{lcl}
\hline Association analysis & $\boldsymbol{r}$ & $\boldsymbol{P}$ value \\
\hline Hepatic and cardiac iron deposition & -0.39 & $0.03^{*}$ \\
Ejection fraction and cardiac iron deposition & 0.33 & 0.08 \\
Serum ferritin and hepatic iron deposition & -0.42 & $0.02^{*}$ \\
Serum ferritin and cardiac iron deposition & -0.25 & $0.04^{*}$ \\
\hline
\end{tabular}

*P value was regarded as statistically significant if $<0.05$ 


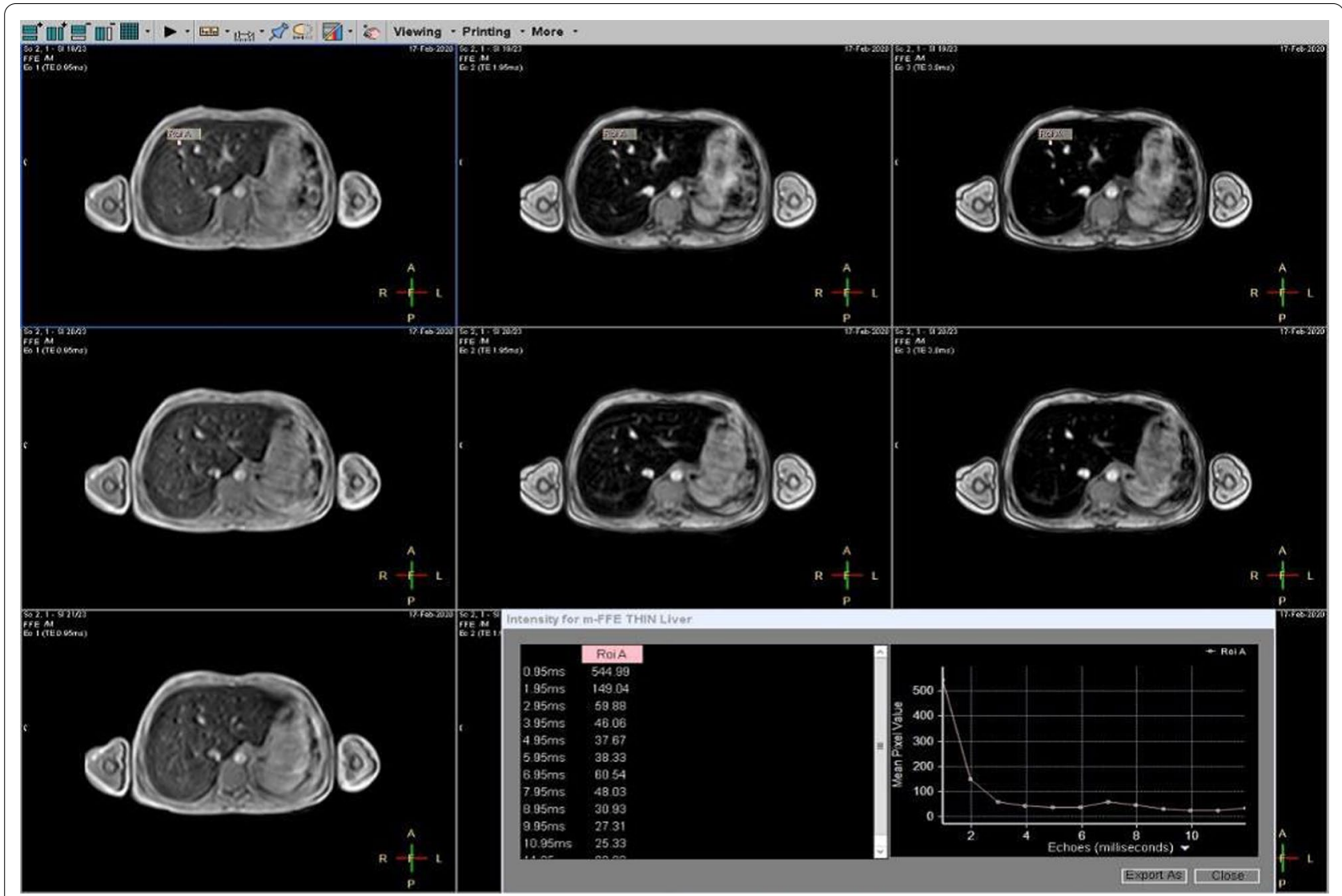

Fig. $2 \mathrm{MRIT2}$ in a 13-year-old male child with thalassemia major demonstrating moderate hepatic iron overload denoted by a dark signal in the liver

This study found that $10 \%$ of participants had severe myocardial iron overload ( $\mathrm{T} 2 *<10 \mathrm{~ms})$, and all cases with severe cardiac iron overload had left ventricular ejection fraction affection. We did not use conventional echocardiography to examine cardiac function as it is an operator and technique dependent that has failed previously to expect heart failure risk in cases with thalassemia. We also have presented that $76.6 \%$ of the cases had a noticeable liver iron overload (LIC 4-13.4 mg/g). The occurrence of severe liver iron overload (LIC $>12 \mathrm{mg} / \mathrm{g}$ ) in our work cases was 33.3\%. Moderate-to-severe hepatic iron inclusion was recorded in more than $50 \%$ of cases. A weak association between heart and liver iron concentration was observed. There was no statistically significant difference in the liver iron T2* levels between those with or without observable heart siderosis.

The same observation was recorded by Anderson et al. [10] and Azarkeivan et al. [24], who stated that there was no association between cardiac and liver iron load. Our study depends on quantitative assessment of $\mathrm{T} 2 *$ value which is the most accurate method for iron load assessment as proved by Azarkeivan et al. [24], who noted that
MRI T2* is an effective, reproducible, and noninvasive approach for determining the iron concentration in tissue, thus avoiding invasive biopsy in some cases.

Additionally, Fragasso et al. [25] agreed that the T2* technique is the most accurate method to detect cardiac damage, even more than electrocardiography and stress echocardiography. Ejection fraction (EF) was determined utilizing a standardized CMR protocol, and $\mathrm{EF}<56 \%$ is regarded as an indicator of heart failure, and with heavy iron deposition and T2* levels below $10 \mathrm{~ms}$, the significant decrease in cardiac function is seen in the form of a decrease in EDV below 56\% and increased ESV. This matches the study results conducted by Meyer et al. [26], which proved that increased cardiac siderosis evaluated by $\mathrm{T} 2 * \mathrm{CMR}$ is linked with LV dysfunction, and this may be a key determinant of cardiac malfunction in thalassemia major cases. Quatre et al. [27] also showed that cardiac $\mathrm{T}^{*}$ values were significantly associated with LVEF dysfunction. In the study of El-Beshlawy et al. [28], it was discovered that the association between heart iron concentration and serum ferritin determined by MRI scans varied in intensity, corresponding to our findings 


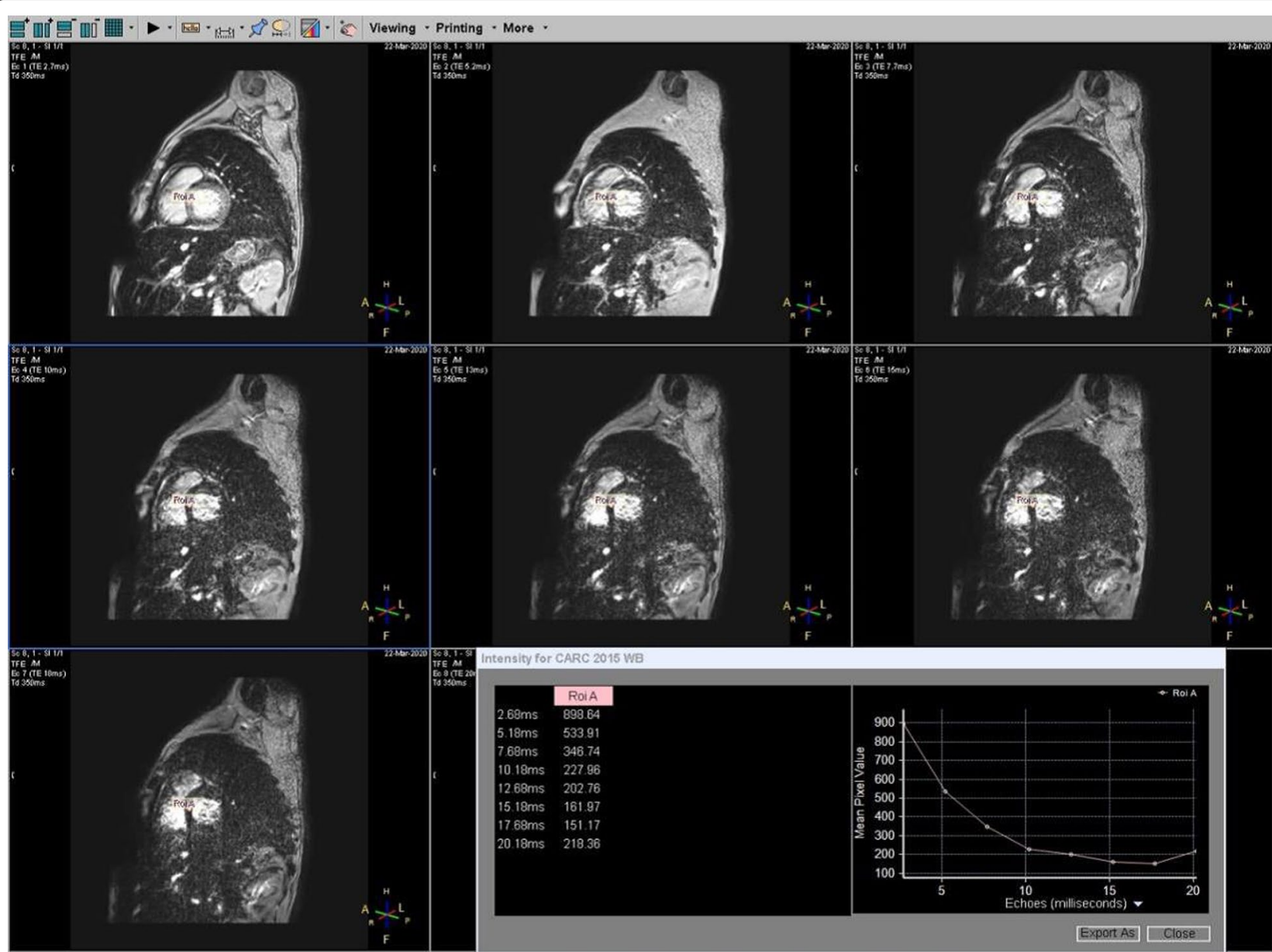

Fig. 3 Left ventricular (LV) short-axis MRI in an 8-year-old male child with TM using gradient-echo sequence with multiple echo times (TEs) for evaluation of T2* ROI is drawn at mid-IVS. Note that the signal intensity of the interventricular septum becomes dark at greater TEs denoting severe iron overload

of a weak association between serum ferritin and cardiac and liver iron loads. With the recent wide availability of MRI systems, it is mandatory to use cardiac MRI in thalassemia cases as a safe, noninvasive and accurate modality for early detection of iron overload and for follow-up after treatment by chelating agents to avoid the inaccurate and invasive technique of liver biopsy for estimation of iron overload.

\section{Conclusion}

Assessment of ventricular function in thalassemia cases is mandatory. Cardiac and hepatic siderosis is common in TDT children and might manifest at a very young age. Cases with cardiac iron overload may progress into cardiomyopathy and heart failure even before developing clinical symptoms. Liver iron and serum ferritin do not associate with the severity of iron overload in the heart. This work supports the fact that the $\mathrm{T} 2 *$ technique is regarded as cardiac iron's gold standard measurement.
We recommend routine monitoring and follow-up of iron overload by using T2* MRI for all TDT children for early detection of any possible complication. Timely treatment could reduce the mortality rate and improve the life quality in these cases.

\section{Study limitation}

This was a single-center study performed on a small number of cases.

\section{Abbreviations}

TDT:Transfusion-dependent B-thalassemia major; LVEF: Left ventricular ejection fraction; LIC: Liver iron concentration; TM: Thalassemia major; DFX: Deferasirox; DFP: Deferiprone; DFO: Desferrioxamine; TCRN: Thalassemia clinical research network.

\section{Acknowledgements}

The authors express their gratitude to all enclosed subjects in this study. 


\section{Authors' contributions}

AME contributed to the design of the study, ASI contributed to the methodology and MRI protocol used in the study, MAE analyzed and interpreted the patient data regarding the MRI findings, ASA have drafted the work and contributed to statistical analysis, AMI contributed to the clinical and laboratory parts of the study and referral of the patients to the radiology department. All authors read and approved the final manuscript.

\section{Funding}

No disclosure of funding received for this work from any organization.

\section{Availability of data and material}

This prospective clinical study was done in the Radiology department at Minia Maternity and Children University Hospital in Egypt. Sixty children diagnosed with transfusion-dependent beta-thalassemia major (TDT) depending on hematological and clinical evaluation and aged between 5 and 15 years were included in the study in the period from February 2020 to February 2021

\section{Declarations}

\section{Ethics approval and consent to participate}

The study was approved by the local ethical committee at Minia university, faculty of medicine. A written informed consent from all patients' parents before the study was filled and signed.

\section{Consent for publication}

All patients included in this research gave written informed consent to publish the data contained within this study by their parent.

\section{Competing interests}

The authors declare that they have no competing interests.

\section{Author details}

${ }^{1}$ Department of Radiology, Faculty of Medicine, Minia Maternity and Children University Hospital, Minia University, Minia, Egypt. ²Department of Radiology, Faculty of Medicine, Ain Shams University, Cairo, Egypt. ${ }^{3}$ Department of Pediatrics, Faculty of Medicine, Minia University, Minia, Egypt.

\section{Received: 30 August 2021 Accepted: 26 October 2021}

Published online: 01 November 2021

\section{References}

1. Weatherall DJ (1996) Oxford textbook of medicine. Anaemia as a world health problem. Oxford University Press, Oxford, pp 3463-3482

2. Brittenham GM (2011) Iron-chelating therapy for transfusional iron overload. N Engl J Med 364(2):146-156. https://doi.org/10.1056/NEJMc t1004810.PMID:21226580

3. Borgna-Pignatti C, Rugolotto S, De Stefano P et al (2004) Survival and complications in patients with thalassemia major treated with transfusion and deferoxamine. Haematologica 89(10):1187-1193

4. Modell B, Khan M, Darlison M (2000) Survival in beta-thalassaemia major in the UK: data from the UK Thalassaemia Register. Lancet 355(9220):2051-2052. https://doi.org/10.1016/S0140-6736(00)02357-6

5. Tanner MA, Galanello R, Dessi C et al (2008) Combined chelation therapy in thalassemia major for the treatment of severe myocardial siderosis with left ventricular dysfunction. J Cardiovasc Magn Reson 10(1):12. https://doi.org/10.1186/1532-429X-10-12.PMID:18298856; PMCID:PMC2289829

6. Olivieri NF, Nathan DG, MacMillan JH et al (1994) Survival in medically treated patients with homozygous beta-thalassemia. N Engl J Med 331(9):574-578. https://doi.org/10.1056/NEJM199409013310903

7. Sado DM, Maestrini V, Piechnik SK et al (2015) Noncontrast myocardial T1 mapping using cardiovascular magnetic resonance for iron overload. J Magn Reson Imaging 41(6):1505-1511. https://doi.org/10.1002/ jmri.24727

8. Davis BA, O'Sullivan C, Jarritt PH, Porter JB (2004) Value of sequential monitoring of left ventricular ejection fraction in the management of thalassemia major. Blood 104(1):263-269. https://doi.org/10.1182/ blood-2003-08-2841

9. Westwood MA, Anderson LJ, Maceira AM et al (2007) Normalized left ventricular volumes and function in thalassemia major patients with normal myocardial iron. J Magn Reson Imaging 25(6):1147-1151. https://doi.org/10.1002/jmri.20915

10. Anderson LJ, Holden S, Davis B et al (2001) Cardiovascular T2-star (T2*) magnetic resonance for the early diagnosis of myocardial iron overload. Eur Heart J 22(23):2171-2179. https://doi.org/10.1053/euhj.2001. 2822

11. Westwood MA, Firmin DN, Gildo M et al (2005) Intercentre reproducibility of magnetic resonance T2* measurements of myocardial iron in thalassaemia. Int J Cardiovasc Imaging 21(5):531-538. https://doi.org/ 10.1007/s10554-005-0651-2

12. Wood JC, Otto-Duessel M, Aguilar M et al (2005) Cardiac iron determines cardiac $\mathrm{T}^{*}, \mathrm{~T} 2$, and $\mathrm{T} 1$ in the gerbil model of iron cardiomyopathy. Circulation 112(4):535-543. https://doi.org/10.1161/CIRCULATIO NAHA.104.504415

13. Ghugre NR, Enriquez CM, Gonzalez I et al (2006) MRI detects myocardial iron in the human heart. Magn Reson Med 56(3):681-686. https:// doi.org/10.1002/mrm.20981.PMID:16888797;PMCID:PMC2887674

14. Tubman VN, Fung EB, Vogiatzi M et al (2015) Thalassemia clinical research network. Guidelines for the standard monitoring of patients with thalassemia: report of the thalassemia longitudinal cohort. J Pediatr Hematol Oncol 37(3):e162-e169. https://doi.org/10.1097/MPH. 0000000000000307

15. Garbowski MW, Carpenter JP, Smith G et al (2014) Biopsy-based calibration of $2^{*}$ magnetic resonance for estimation of liver iron concentration and comparison with R2 Ferriscan. J Cardiovasc Magn Reson 16(1):40. https://doi.org/10.1186/1532-429X-16-40.PMID:24915987; PMCID:PMC4064805

16. Carpenter JP, He T, Kirk P et al (2011) On T2* magnetic resonance and cardiac iron. Circulation 123(14):1519-1528. https://doi.org/10.1161/ CIRCULATIONAHA. 110.007641

17. Di Tucci AA, Matta G, Deplano S et al (2008) Myocardial iron overload assessment by $\mathrm{T}^{*}$ magnetic resonance imaging in adult transfusion dependent patients with acquired anemias. Haematologica 93(9):1385-1388. https://doi.org/10.3324/haematol.12759

18. Wood JC, Enriquez C, Ghugre N et al (2005) MRI R2 and R2* mapping accurately estimates hepatic iron concentration in transfusiondependent thalassemia and sickle cell disease patients. Blood 106(4):1460-1465. https://doi.org/10.1182/blood-2004-10-3982

19. Neufeld EJ (2006) Oral chelators deferasirox and deferiprone for transfusional iron overload in thalassemia major: new data, new questions. Blood 107(9):3436-3441. https://doi.org/10.1182/blood-2006-02002394.PMID:16627763

20. The Cooley's Anemia Foundation. Position statement on MRI based hepatic iron assessment methods. http://www.thalassemia.org/2012position-statement-on-mri-based-hepatic-iron-assessment-methods-2. Accessed 12 May 2021

21. Macarini L, Marini S, Pietrapertosa A et al (2005) Non cardiopatic and cardiopatic beta thalassemic patients: quantitative and qualitative cardiac iron deposition evaluation with MRI. Radiol Med 109(1-2):77-90 (English, Italian)

22. St Pierre TG, Clark PR, Chua-anusorn W et al (2005) Noninvasive measurement and imaging of liver iron concentrations using proton magnetic resonance. Blood 105(2):855-861. https://doi.org/10.1182/ blood-2004-01-0177

23. Tanner MA, Galanello R, Dessi C et al (2007) A randomized, placebocontrolled, double-blind trial of the effect of combined therapy with deferoxamine and deferiprone on myocardial iron in thalassemia major using cardiovascular magnetic resonance. Circulation 115(14):18761884. https://doi.org/10.1161/CIRCULATIONAHA.106.648790

24. Azarkeivan A, Hashemieh M, Akhlaghpoor S et al (2013) Relation between serum ferritin and liver and heart MRI T2* in beta thalassaemia major patients. East Mediterr Health J 19(8):727-732

25. Fragasso A, Ciancio A, Mannarella C et al (2011) Myocardial iron overload assessed by magnetic resonance imaging (MRI)T2* in multi-transfused patients with thalassemia and acquired anemias. Eur J Intern Med 22(1):62-65. https://doi.org/10.1016/j.ejim.2010.10.005 
26. Meyer P, Filippatos GS, Ahmed Ml et al (2010) Effects of right ventricular ejection fraction on outcomes in chronic systolic heart failure. Circulation 121(2):252-258. https://doi.org/10.1161/CIRCULATIONAHA.109. 887570

27. Quatre A, Jacquier A, Petit P et al (2014) MRI monitoring of myocardial iron overload: use of cardiac MRI combined with hepatic MRI in a cohort of multi-transfused patients with thalassaemia. Diagn Interv Imaging 95(11):1065-1069. https://doi.org/10.1016/j.diii.2014.01.007
28. El Beshlawy A (2005) The Egyptian experience with oral iron chelators Hematology 10(Suppl 1):174-175. https://doi.org/10.1080/1024533051 2331390302

\section{Publisher's Note}

Springer Nature remains neutral with regard to jurisdictional claims in published maps and institutional affiliations.
Submit your manuscript to a SpringerOpen ${ }^{\odot}$ journal and benefit from:

- Convenient online submission

- Rigorous peer review

- Open access: articles freely available online

- High visibility within the field

- Retaining the copyright to your article

Submit your next manuscript at $\boldsymbol{\nabla}$ springeropen.com 